\title{
fMRI: a tree with fuzzy roots
}

\author{
A deeper understanding of the mechanisms underlying functional magnetic resonance imaging \\ signals is crucial for maximizing the return on human fMRI research.
}

Since 2010, the US National Institutes of Health (NIH) has awarded over $\$ 200$ million on research related to functional magnetic resonance imaging (fMRI) (according to the 'NIH RePORTER' database) - a number that has been increasing over the last years.

Despite the increased interest in this technology and the huge investments, it's striking how little we know about the fMRI signal itself, and in particular about how to interpret it in terms of brain activation. The incomplete understanding of this signal limits the inferences that can be made from fMRI data and thus their usefulness for understanding how our brains work. It is time to focus resources and scientific effort on determining the fine neurophysiological details underlying these signals.

The vast majority of fMRI studies are based on the blood oxygen level-dependent (BOLD) contrast. BOLD signals reflect changes in blood flow, blood volume and the metabolic rate of oxygen consumption in the local tissue. Since the 1890s, it has been known that changes in the blood are closely linked to neural activity. fMRI studies use this connection to make assumptions about how the brain is functionally organized.

Understanding the functional organization of the brain has preoccupied scientists for centuries. Electrophysiological methods reveal the activity of cells in fine detail and can be used to study the function of relatively small brain circuits. But in order to understand how our minds work, the activity of the brain must also be studied at a much larger scale. Technologies like fMRI, among others, allow the non-invasive examination of processes related to neural activity throughout the entire brain.

The strong growth in government funds (and scientific publications) allocated to fMRI-based studies might in part be explained by the availability of MRI scanners in many medical institutions. The vast majority of this funding has been given to projects studying cognition, functional connectivity or neurological diseases in humans.

In a typical fMRI experiment, blood oxygen level changes in the brain are monitored while a subject is exposed to a particular stimulus, performs a task or simply rests. This type of research yields correlative information about regions in the brain that are associated with the task, stimulus or state in question. However, what these data can tell us about the mechanisms underlying human cognition or neurological disease depends on our understanding of the specificity and spatio-temporal resolution of the BOLD signal itself. What, in other words, is really happening in a brain region that 'lights up' in BOLD fMRI?
Simultaneous fMRI and electrophysiological recordings in animal models have shed some light on the types of electrical signals that elicit a BOLD response, and it is now accepted that the signal represents activations of neuron ensembles. However, it is unclear which types of neurons elicit this signal and how their different temporal activation patterns affect it. It's also unclear how excitation-inhibition balances in the circuits and higher-order neuromodulation events influence the BOLD signal. The role played by astrocytes - which serve as bridges between neurons and the vasculature in the brain-in modulating the BOLD response has also remained a mystery.

Answering these questions will give us a better handle on the types of inferences that can be made from fMRI data, in both quantitative and qualitative terms. Moreover, a better understanding of the biology underlying the signal will influence the design of future experiments and maximize the potential of the technique.

But how will these questions be answered?

Work in animal models using multimodal technologies in conjunction with studies in humans will be key. The combination of fMRI with optogenetic stimulation of genetically defined neurons in animal models, as recently shown, has enabled scientists to study the causality between the activation of specific neuronal populations and the BOLD signal.

In this issue of Nature Methods, Fritjof Helmchen and colleagues combine fMRI with fiber optic-based calcium imaging of neuronal and glial activity and uncover a glial contribution to the BOLD response (Article p597; News \& Views p547). Both of these approaches offer unprecedented possibilities for performing cellular-level resolution studies of the relationship between 'brain activation' and the BOLD signal. If applied in a systematic manner throughout the brain in animal models, such high-resolution mapping should yield valuable information for human studies; however, little such research is being done.

Funding of projects focused on gaining a deeper understanding of the BOLD signal should be given a higher priority. Animal MRI scanners are not as ubiquitous as their human-oriented counterparts, so this will not be cheap. The studies will also require different experimental approaches and collaboration between neuroimagers and cellular neuroscientists. Researchers trained in multidisciplinary areas may be best poised to tackle this problem-possibly one of the most important and exciting scientific challenges of our time. 